\title{
Simulación por ordenador y enseñanza de la Física $^{1}$
}

\author{
Manuel Belmonte y José L. Rodríguez Illera
}

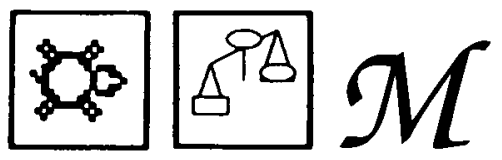

Queremos presentar una reflexión general sobre el uso de determinados tipos de programas informáticos en la enseñanza, a la vez que el uso concreto que uno de ellos ba experimentado durante los últimos años (1991-95) en un instituto de bacbillerato. Se trata, si se quiere, de un estudio de casos, si bien expuesto desde el ángulo del propio profesor implicado.

\section{LA SIMULACION POR ORDENADOR}

El llamado «software educativo» es de muchos tipos y ha sido objeto de numerosas clasificaciones. Una de las ideas con más fuerza para pensar sus potenciales usos educativos es la de considerar el grado de apertura que tiene - considerando por «apertura» su formato modificable por el usuario final. Al señalar (King, 1990) la apertura como criterio relevante se tiende a desplazar la problemática del «buen» software educativo de las consideraciones informáticas o/y instructivas a las formas de utilización del programa. En realidad, creemos que la apertura es verdaderamente importante cuando los contenidos y la interacción presente en el programa son de interés para fines educativos, por lo que se trata de un criterio estrechamente unido a las «funciones» (contenidos, procedimientos implicados, etc) que el software realiza. Por tanto, los criterios sobre los tipos de software conllevan pensar si se trata de un programa tutorial, de una simulación, de un hipertexto, de un tutor inteligente, de un programa de ejercitación, etc, además de si tiene o no una estructura abierta y en qué grado. $\mathrm{Y}$, desde luego, sin pensar que sólo los programas abiertos son útiles.

La experiencia que vamos a referir se ha realizado con un programa abierto que es, además, un entorno de simulación. Todo programa de simulación es mínimamente abierto por cuanto permite al usuario variar algunos datos o/y parámetros de control de la simulación. Sin embargo, el caso que presentamos supone un tipo de simulación con características que lo hacen especialmente adaptado para su uso escolar. El programa se denomina Interactive Physics, y puede esquematizarse así:

- es un entorno de simulación (esto es, permite realizar diferentes pruebas de simulación) dentro de la enseñanza de la Física. El tipo de áreas curriculares que cubre son, aproximadamente, las enseñanzas de Mecánica del actual B.U.P. y C.O.U., pudiendo extenderse incluso a primeros cursos de docencia universitaria. 
- las simulaciones que pueden organizarse por parte del profesor y alumnos son ilimitadas. Cada simulación consiste en el diseño de uno o varios móviles (construídos mediante formas poligonales), de una situación espacial entre ellos (planos, objetos fijos, etc) y la aplicación de unas fuerzas que determinarán su movimiento. Una vez diseñada la simulación, se ejecuta: los móviles se mueven en función del resto de objetos y fuerzas de la situación.

- las simulaciones son siempre visuales. El movimiento se ve en la pantalla del ordenador y es una representación (idealizada como toda representación) de los movimientos reales de los cuerpos. La representación es «realista» en el sentido de describir las trayectorias que las leyes de la Física prescriben (gráficos 1 y 2). Factores generales como la fuerza de la gravedad, el rozamiento o la elasticidad pueden ser variados globalmente y afectan a la trayectoria de los objetos implicados.
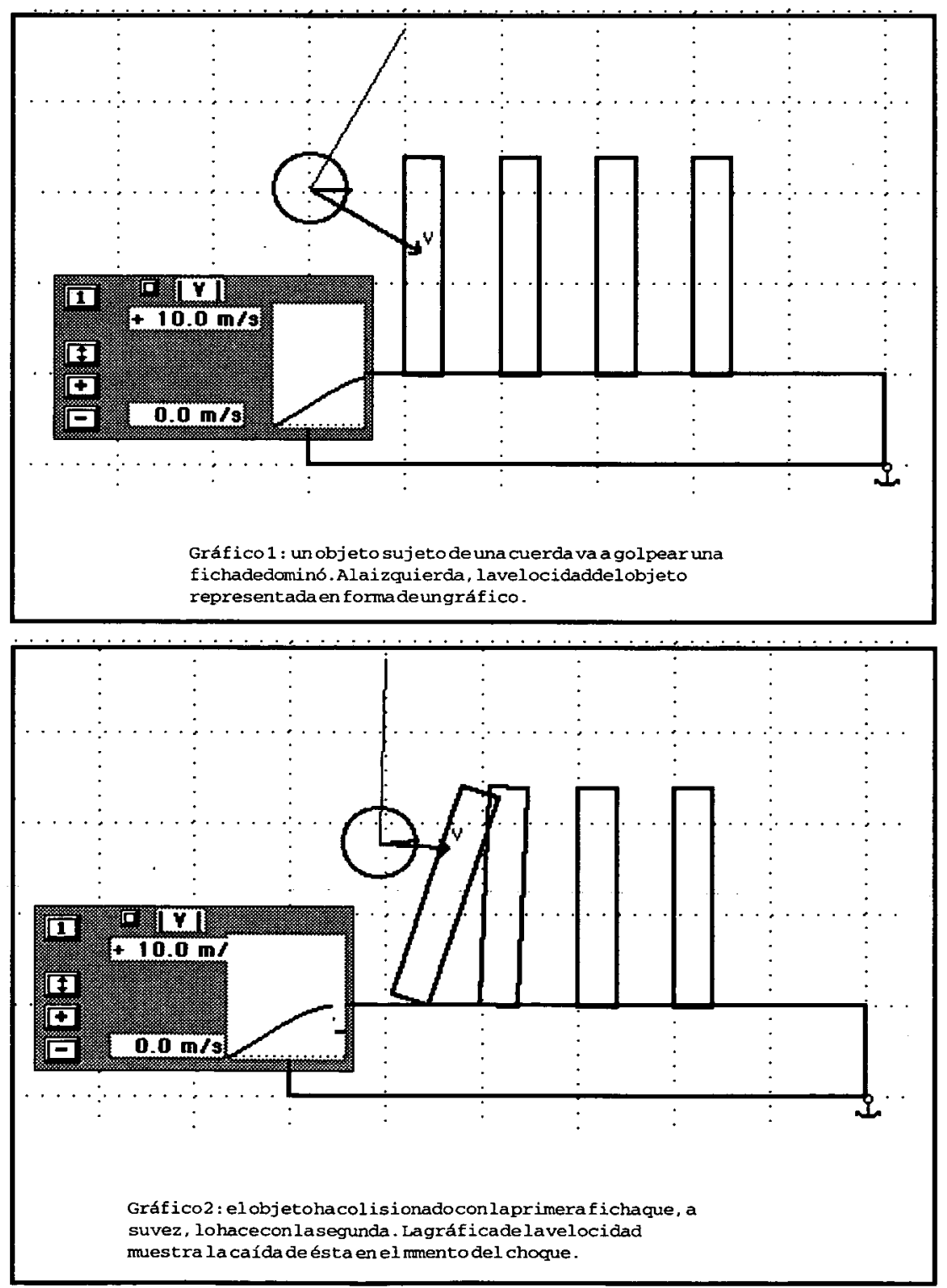
- el alumno puede obtener datos númericos o gráficos de un buen número de variables implicadas (velocidad, aceleración, rotación, posición, momento angular, etc). Los simuladores de los aparatos de medida de estos datos pueden verse en pantalla de manera simultánea a la ejecución de la simulación (gráfico 3). Los datos obtenidos pueden ser trasvasados fácilmente a una hoja de cálculo para su análisis posterior (gráfico 4).
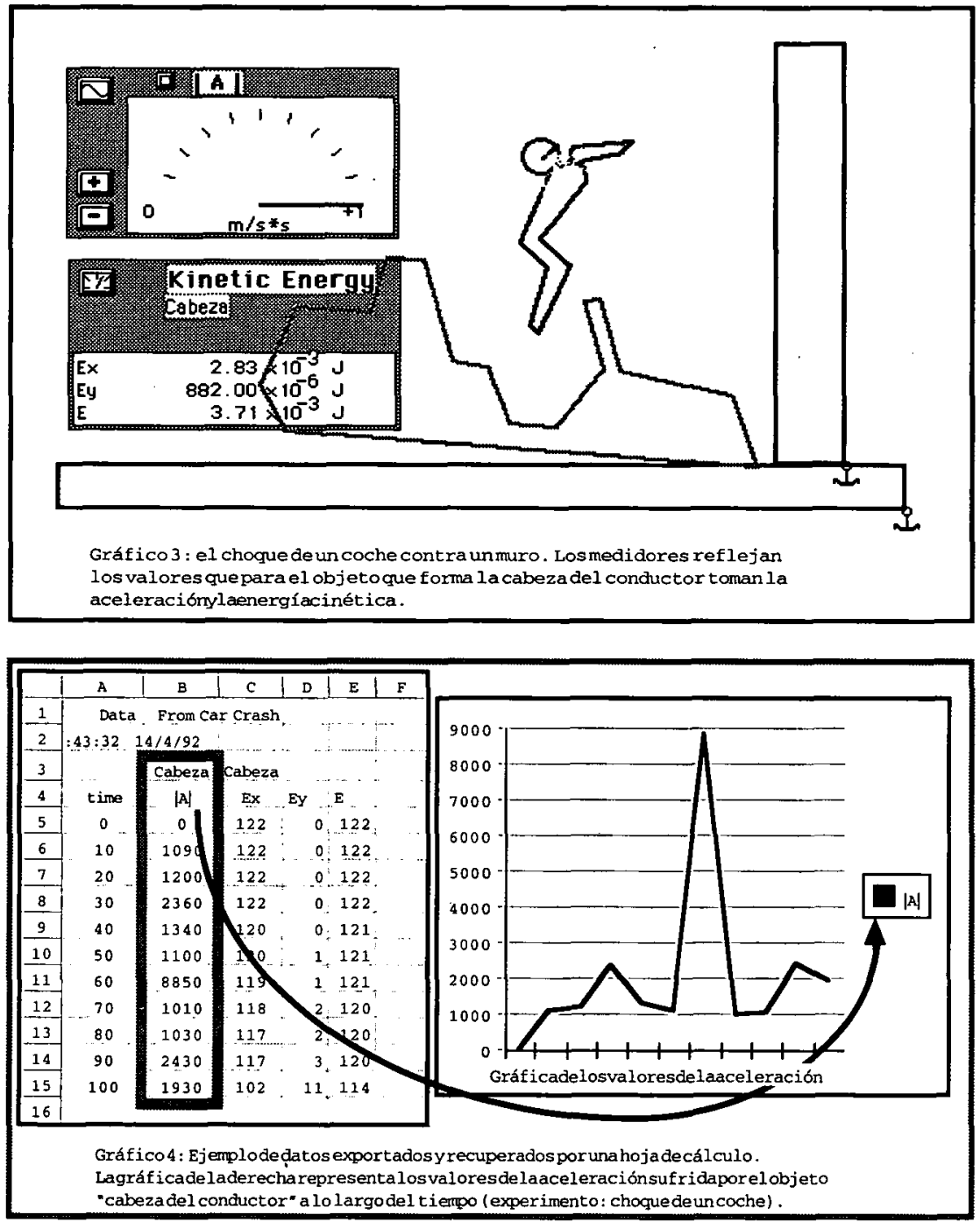

- finalmente, el desarrollo animado de la simulación queda registrado en la memoria del ordenador, pudiendo ser tratado como una cinta de vídeo: parándolo, acelerándolo, volviendo atrás, etc.

En conjunto, se trata de un entorno de simulación muy poderoso a la vez que muy circunscrito a su dominio. La posición del profesor o del alumno cuando lo utiliza es la de poseer un control muy elevado sobre un número de parámetros importante, con un interfaz de uso inmediato basado en el ratón y teclado. Las limitaciones del entorno responden a las limitaciones/simplificaciones en la enseñanza de la Física 
a los niveles educativos mencionados. Las esquematizaciones en cuanto a la representación gráfica y animada de los objetos, fuerzas y movimiento responden a las convencionales en la enseñanza secundaria y universitaria (con alguna pequeña excepción).

Las características formales de este entorno permiten que la actividad del usuario sea semejante a la descrita idealmente por De Hoog et al (1991) en los aspectos permitidos por el modelo/entorno de simulación. La descripción de De Hoog et al. es muy exhaustiva —desde el punto de vista analítico- cuando se refiere a otros aspectos del interfaz de simulación, por lo que no todos ellos pueden estar presentes en un único entorno. Conviene señalar que el caso de las simulaciones de procesos físicos permite un grado de "verosimilitud» mucho mayor que el de procesos más abstractos, menos ligados a una base física real. Esto es así por la continuidad existente entre el modelo subyacente a la simulación y la representación gráfica que puede hacerse de la misma: la Física moderna se ha construído siempre como una representación en un espacio cartesiano (otra cuestión sería intentar representar modelos de mecánica cuántica...). Sin embargo, cualquier representación no deja nunca de tener un aspecto claramente convencional en cuanto a la forma misma de la representación y su relación con lo representado. En el caso de este programa, la forma de la representación de los valores que controlan los parámetros de la simulación para cada móvil (masa, velocidad, fricción y elasticidad) pueden ser controlados de manera numérica o/y mediante una escala analógica, mientras que la representación de los resultados se realiza de una forma gráfica (o numérica, a elección del usuario). El grado de similitud que hay entre la representación y el modelo es el suficiente para la convencionalidad representativa de la enseñanza de la Física — por otra parte, es dudoso que representaciones más artificialmente «realistas» como las que provienen de la tecnología del denominado «cyberespacio» o 'realidad virtual' permitan captar mejor los modelos subyacentes a una simulación.

Por otra parte, la utilización de entornos de simulación con fines instructivos es muy poco frecuente en nuestro país. Muy pocas experiencias se han realizado con juegos de ordenador (quizás la principal fuente de simulaciones), limitándose la mayoría a las «simulaciones» que pueden realizarse con una hoja de cálculo o con pequeños programas escritos en un lenguaje de prógramación. Las características descritas de Interactive Physics, y, en especial, la expresión gráfica y animada del resultado del modelo, así como el interfaz de usuario, creemos que lo hacen particularmente adecuado para su uso docente ${ }^{2}$.

De hecho, muchas de las consideraciones sobre las ventajas potenciales de las simulaciones se han hecho pensado en distintos tipos de entornos de simulación, con distintos objetivos, con distintos tipo de modelos, y, sobre todo, con distintos interfaces tanto del modelo como del usuario.

El argumento común subyacente es pensar que las simulaciones constituyen un entorno donde la experimentación, el proceso de ensayo y error, puede realizarse rápida y eficazmente, y que, por tanto, el ciclo de razonamiento se corrige con mayor facilidad. Todos los modelos sobre el razonamiento o/y acción humana (desde el clásico TOTE de Miller et al. (1960), hasta los revisados por Goodyear et al. (1991), incluyen una fase fundamental de prueba o experimentación), por lo que el gran interés de los entornos de simulación estriba en ofrecer una representación inteligible del modelo subyacente al proceso que se modeliza, en la que aparecen claramente las variables sobre las que es dado experimentar. Nuestra idea, sin embargo, es que tales modelizaciones no siempre adoptan un formato representacional adecuado para su comprensión (o que no siempre es posible adoptarlo), y que cada entorno de simulación será distinto en la facilitación para el estudiante tanto de los procesos de experimentación como de las mejoras cognitivas asociadas. 


\section{DE LA TEORIA A LA PRACTICA: ALGUNAS VALORACIONES}

En esta perspectiva, se ha empezado un proceso de experimentación del programa en situaciones reales de enseñanza. Durante el curso 1991-92, un Instituto de Bachillerato de Barcelona ha dispuesto de un ordenador adecuado para el uso del programa (con conexión y pantalla de cristal líquido), así como una formación mínima sobre su funcionamiento. Los profesores implicados han diseñado ejercicios para ser insertos en momentos concretos de su curso, con el objetivo de apoyar en el aprendizaje de nociones difíciles para sus estudiantes. El programa se ha utilizado (cfr. 3) como ayuda en las exposiciones, a través de la proyección de la pantalla del ordenador, o bien como simulación «realista» de ejercicios que previamente se habían intentado resolver con «lápiz y papel».

Aunque es evidente que no puede pretenderse un tipo de evaluación exhaustiva, sí que merece la pena recoger tanto las impresiones de los profesores y alumnos, como las condiciones mismas de la experiencia. Creemos que apuntan en la dirección señalada sobre el valor de las simulaciones y, específicamente, sobre las ventajas representacionales que ofrece este programa.

\section{La valoración de los profesores}

Los profesores han valorado tres aspectos diferentes: la facilidad de uso, la potencia del programa y su utilidad instructiva. Todos han encontrado que el programa es muy fácil de utilizar y que sólo requiere unas pocas horas de adaptación. Este es un aspecto muy importante: de hecho dos profesores empezaron a utilizar el programa a raíz de ver cómo otro profesor lo hacía, y en ningún momento la forma de operar supuso una barrera o una dificultad importante. Los programas de simulación tienen asociada una cierta aura de dificultad, que es necesario romper a través de un diseño del interfaz de usuario muy cuidado, que además de gráfico delimite muy bien el número de elementos que entran en la simulación y la facilidad para reunirlos en forma de modelo3.

La potencia del programa es también otro aspecto destacado por los profesores, que ven en él la posibilidad para completar experiencias prácticas realizadas de forma "real", a la vez que para transformar esas mismas prácticas en ejercicios cuantitativos y precisos. Pero lo que, sin duda, resulta más interesante son las consecuencias que los profesores ven para sus alumnos: en primer término, su capacidad de motivación generada por la posibilidad de comprobación de las predicciones o de los resultados que los alumnos han alcanzado por otros métodos. También, la ayuda que el programa aporta a la eliminación de "preconceptos» erróneos en los alumnos, y en la adquisición de los conceptos correctos que los sustituirán.

En general, se podría decir que los profesores implicados en la experiencia han valorado de manera muy positiva tanto el programa (fácil de usar, potente) como sus implicaciones didácticas (motivación generada, cambios cognitivos). Debe decirse que se trata de profesores muy comprometidos con la enseñanza de su materia pero ésto no es inhabitual.

\section{La valoración de los alumnos}

Los alumnos implicados han realizado una valoración diferente, aunque no por ello menos positiva. Algunos aspectos del programa creen que podrían mejorarse: la falta de color (en realidad, se utilizó una pantalla de cristal líquido que sólo funcio- 
naba en blanco y negro), el estar en inglés, el realismo de los objetos —que son siempre polígonos o círculos-, etc.

Creemos que se trata de apreciaciones muy justas, a excepción de la relativa al "realismo" de los objetos. Representaciones más realistas conllevarían una complejidad mucho más alta y dificultarían la dinámica de los objetos. Por otra parte, quizás el referente implícito de comparación son los vídeojuegos que son simulaciones de conducción o de aventuras; si así fuese, es evidente que el objetivo es muy distinto: la esquematización del objeto y de las relaciones con otros objetos es, en el caso, de un programa instructivo una virtud que apunta más al modelo subyacente que al realismo visual de la situación. En cualquier caso, se trata de una cuestión abierta: quizás representaciones más detalladas conllevasen una motivación mayor en la utilización inicial del programa.

Otro de los aspectos valorados por los alumnos es su propio proceso de aprendizaje. En general, afirman que el programa les permite eliminar dudas y completar los conocimientos adquiridos en las prácticas. Todos valoran el ahorro de tiempo que supone, a la vez que colocan en primer término la importancia de realizar las prácticas en antes de utilizar el ordenador. Una característica que les parece muy útil es la capacidad de guardar en memoria la animación-simulación creada, y poder «rebobinar» hacia atrás con facilidad.

\section{COMO SE HA HECHO: UNA METODOLOGIA}

Durante los cuatro años en que se ha experimentado la aplicación de este programa en un aula de secundaria, en segundo y tercero de B.U..P., el programa ha sido empleado para diferentes actividades dentro del desarrollo de una Unidad Didáctica de física y química diseñada según las premisas del aprendizaje significativo, es decir, acomodada a un modelo constructivista de la enseñanza de las ciencias experimentales. Es importante destacar que la utilización de la herramienta informática se ha inscrito en la problemática más general de la unidad y no al revés.

En ese marco, la organización de la Unidad Didáctica ha sido la siguiente (nos referiremos más adelante a estos momentos por sus números):

1. Se iniciaba por una evaluación inicial, donde el alumnado exponia sus preconceptos sobre un tema concreto de física.

2. Seguidamente, las experiencias en el laboratorio, diseñadas para que se relacionaran con la evaluación inicial y crearan conflictos al entrar en contradicción con los preconceptos expuestos en la evaluación inicial.

3. Confección de UVE heurísticas sobre las experiencias.

4. Consulta bibliográfica o debate o trabajo en grupo de seis personas.

5. Aplicación de los nuevos conocimientos, del nuevo paradigma a la resolución de situaciones cotidianas problemáticas o a la interpretación y explicación de dichas situaciones de acuerdo con la nueva concepción.

6. Pequeñas investigaciones en casa sobre fenómenos acaecidos en el entorno habitual y diario del alumnado.

El software de simulación ha mostrado ser de una gran utilidad en todos los apartados anteriores, excepto el primero, donde, evidentemente, a priori se descartó su empleo, y en el tercero, que corresponde a una labor inductiva y de tabulación de datos del alumnado. A continuación indicaremos cómo ha contribuido a la mejora del rendimiento y a una mejor comprensión, significatividad y operatividad de los conceptos impartidos.

En (2), los alumnos desarrollaban primero su actividad en el laboratorio, de una forma inductiva y cooperando entre ellos. Con ello entraban en contacto directo, sensorial, con el fenómeno o realidad a investigar. De esta forma se obtenían unos 
datos cualitativos o semicuantitativos. Por ejemplo, en el análisis de los choques, los alumnos observaban los choques de carritos moviéndose por unos rieles. Las masas y las velocidades de los carritos variaban de forma controlada. Todas las personas podían observar cómo algo se intercambiaba en estas colisiones. Era la conclusión cualitativa, pero les era imposible inducir ley alguna al no poder medir con precisión las velocidades antes y después de los choques. La causa de esta imposibilidad de lograr una medición aceptable eran dos:

a) la inexperiencia del alumnado. Hay que tener en cuenta que no son cronometradores oficiales, sino adolescentes que se inician en las ciencias experimentales.

b) La falta de medios adecuados en un laboratorio de enseñanza secundaria. No se trata de un laboratorio universitario ni de investigación.

Aquí es donde el software puede ayudar. Una vez familiarizados con el problema y obtenidos los datos anteriores, cualitativos o semicuantitativos, los alumnos repetían las mismas experiencias en el ordenador. Con ello obtenían datos cuantitativos que le permitian inducir leyes empíricas, como por ejemplo, la de la conservación de la energía cinética en los choques elásticos o la de la conservación de la cantidad de movimiento en cualquier choque, elástico o no.

$\mathrm{Y}$, aún más importante, podían realizar la misma experiencia en marcos diferentes totalmente imposibles de conseguir en una experiencia real. Por ejemplo, en el caso anteriormente citado de los choques, se podía realizar

- En el plano, en direcciones perpendiculares o según un cierto ángulo.

- Con gravedades diferentes.

- Con diferentes rozamientos entre el plano y los cuerpos que chocaban.

- Con total ausencia de rozamiento.

- En el vacio o con aire de densidades diferentes.

- En estado de ingravidez.

En este apartado, los alumnos se involucraban en gran manera, sugiriendo nuevas variantes que correspondían a preconceptos personales que querían comprobar. Por ejemplo, en el caso anterior, en los dos cursos escolares en que se llevó a cabo la experiencia, se sugirió la posibilidad de cambiar el volumen sin cambiar las masas.

Es importante remarcar que es imprescindible desarrollar primeramente la experiencia de forma manual en el laboratorio antes de simularla en el ordenador. En caso contrario, los alumnos pierden la necesaria conexión con la realidad y la simulación resulta poco significativa para ellos al no poder relacionarla con nada vivencial experimentado anteriormente. Si los alumnos no identifican y reconocen en todo aquello que ven en la pantalla algo que han 'tocado' anteriormente no le confiere la categoría de experiencia, de realidad. Pasa a ser un videojuego donde todo es posible y donde nada tiene porqué comportarse de forma real.

En (4) mientras desarrollaban la actividad, se encontraban con dudas, objeciones opiniones contrapuestas. Al desear dilucidarlas, siempre se les encaminaba hacia la realización de una pequeña prueba o experiencia, real o simulada. Por esta causa, el ordenador se encontraba en el aula, con el programa funcionando. En cualquier momento una persona podia levantarse de su asiento y, en un principio con la ayuda del profesor, pero más tarde sola, plantear la situación en conflicto en la pantalla y decidir la solución adecuada.

Por ejemplo, en el caso del estudio del movimiento parabólico, varios estudiantes de tercero de B.U.P. se encontraron con dificultades para comprender la exposición del texto sobre la constancia de la componente horizontal de la velocidad y la variación de la componente vertical. Algo que en años anteriores les habia ocupado prácticamente una hora de esfuerzo personal sin llegar a captar, a aprehender totalmente la complejidad de la situación, se solventó en estos dos años, con la visión del fenómeno en la pantalla durante unos diez minutos en total, incluido el tiempo 
dedicado al diseño de la simulación. Además, se fueron a su sitio con una página impresa con la variación de las dos componentes de la velocidad en diferentes puntos de la trayectoria, una gráfica posición/tiempo, otra velocidad/tiempo y otra aceleración/tiempo que se dedicaron a analizar y comparar entre sí y con el dibujo del cuerpo en su trayectoria.

La resolución de "problemas» del apartado (5), ha resultado especialmente beneficiada con la utilización de la simulación en el ordenador. Ello ha permitido que:

- Los problemas sean mucho más reales.

- Los alumnos adquieran la capacidad de criticar los resultados obtenidos.

- Los alumnos asuman que están resolviendo casos mediante la aplicación de los nuevos conocimientos, que no se trata de un ejercicio puramente académico sin conexión con la realidad.

- Un reforzamiento inmediato de los conocimientos tanto si la solución ha sido correcta como si no.

- Una mejor comprensión de los conocimientos adquiridos.

Tal vez la mejor forma de exponerlo sea mediante un ejemplo. Supongamos que se plantea el típico problema de indicar qué ángulo y celeridad iniciales debe tener un balón para sobrepasar un muro de cierta altura que tiene delante y del que dista una cierta distancia. Tras un cierto tiempo dedicado al cálculo, algunos alumnos consiguen unos ciertos valores para las magnitudes pedidas. Muchos manuales de pedagogía insisten en la necesidad de una corrección inmediata para lograr un reforzamiento positivo. Dado que es imposible confirmar o negar la bondad de la solución conseguida a medida que cada grupo la alcanza, se opta frecuentemente por proporcionarla de forma que se realiza una autocorrección comparando con la modelo suministrada. Nosotros hemos optado por inducir a los alumnos a introducir sus resultados en la simulación de la situación en la pantalla y hacer correr el programa, con lo que de forma vivencial, animada e incontrovertible se observa si la solución es la adecuada o no. En este caso, como en otros muchos, además el problema se hizo real. El balón llegaba hasta la altura del muro, pero se quedaba encima de él. ¡El muro tenía un ancho! ¡No era la linea sin anchura que muchos problemas académicos suponen! Los estudiantes rehicieron los cálculos teniendo en cuenta el ancho del muro. Pero volvieron a fallar. ¡Tampoco habían tenido en cuenta el diámetro del balón! ¡Este no era el punto másico que también suponen muchos problemas académicos! Finalmente el problema se solucionó y el balón superó el muro. Y el alumnado superó la barrera que separaba la solución idealizada, irreal del problema con la algo más real que consiguió, por que les quedó la duda de qué hubiera pasado si tuvieran en cuenta el rozamiento del aire. Pero para este punto no les quedó tiempo. Pero este detalle no enturbió el hecho de haber conseguido que la cinemática del movimiento parabólico atravesara la puerta de la clase y se fuera con ellos por el mundo.

En cuanto al sexto y último apartado (6), casi no hubiera sido posible sin su concurréncia. Cada equipo de estudiantes realizaba una pequeña investigación como, por ejemplo, el estudio del movimiento de una cometa y de las fuerzas que la mantenían en el aire. Tras el estudio teórico de las fuerzas que suponían que intervenían, diseñaban un programa para simular el vuelo de la cometa. Y lo ejecutaban con los valores calculados. El programa les obligó a modificar mucho todo su planteamiento hasta conseguir mantener en vuelo simulado a su cometa. Ello modificó substancialmente su conocimiento de las fuerzas y de su comportamiento en un caso real cotidiano. Más tarde acabaron su estudio, mediante la construcción de un pequeño túnel de viento, donde hicieron volar una maqueta a escala.

Otros equipos estudiaron otros fenómenos diferentes, utilizando todos ellos en su segunda fase la simulación mediante el programa de ordenador. 


\section{CONCLUSIONES}

Si tuviésemos que establecer unas conclusiones provisionales de esta experiencia, se decantarían por la importancia de poder encontrar herramientas adecuadas para usos instructivos y por su fácil inclusión curricular. No siempre es posible esta doble condición: mucho software educativo sigue siendo de calidad baja, y sus aplicaciones educativas han sido poco o mal previstas por los programadores o diseñadores. Algunos programas de simulación (y de otros tipos) permiten, sin embargo, mostrar la posible dimensión de la informática educativa: facilidad de uso, potencia relativa a la modalidad informática de los contenidos, mejoras cognitivas para los alumnos, mejoras en la motivación ante temas difíciles, etc Es en este camino como se entiende la potencialidad y optimismo mostrada por Salomon et al. (1991) y por tantos otros autores. El uso del ordenador en la enseñanza depende del uso que el profesor o/y los alumnos dan a determinados programas bien diseñados. Es ya hora de que esos programas no sean la excepción sino la norma.

\section{Notas}

' Partes de este artículo fueron expuestas en el el congreso europeo sobre Tecnologías de la Información en la Educación. Una visión crítica. (Barcelona, Octubre de 1992). Dado que la experiencia que se relata ha continuado desde entonces, hemos ampliado considerablemente las referencias a la aplicación curricular.

2 En relación a la exhaustiva caracterización instructiva de las simulaciones que realizan Reingeluth y Schwartz (1989), se puede decir que el programa está dedicado, en sus aspectos de contenido, a la enseñanza de relaciones causales. Las relaciones causales exigen una simplificación en muchos casos, para no dotar al programa de una sobrecarga informativa /cognitiva, además de ser aptas para una relación de búsqueda por parte del estudiante.

${ }^{3}$ No todos los tipos de entorno de simulación permiten una facilidad extrema de utilización. Aquellos que son, por la propia naturaleza de los procesos a modelizar, más abstractos, pueden beneficiarse de un interfaz gráfico pero eso no soluciona la complejidad de la dinámica modelada (como, p.e., el entorno Stella). Cuanto más general es un entorno de simulación más difícil es su utilización para fines detallados.

\section{Referencias}

DE HOOG, R.; DE JONG, T., y DE VRIES, F. (1991). Interfaces for instructional use of simulations. Education \& Computing, vol 6,3-4,359-386.

GOOdYEAR, P.; NJOO, M.; HJINE, H., y VAN BerkUM, J. J. A. (1991). Leaming processes, learner attributes and simulations. Education \& Computing, vol 6, 3-4, 263-304.

King, D. (1990). L'aplicacio del software en l'Educació Especial. En J.L. Rodríguez Illera (comp), Informàtica i Educació Especial. Barcelona: ICE de la Universitat de Barcelona, pp. 10-31.

Miller, G. A.; Gallanter, y Pribram, K. (1960). Plans and the structure of behavior. Nueva York: Holt.

Reigeluth, C. M., y SCHWARTZ, E. (1989). An Instructional Theory for the Design of Computer-Based Simulations. Journal of Computer-Based Instruction, vol 16, 1, 1-10.

SALOMON, G; PERKINS, D. N., y GloberSON, T. (1991). Coparticipando en el conocimiento: la ampliación de la inteligencia humana con las tecnologías inteligentes. Comunicación, Lenguaje y Educación, 13, 6-22. 


\section{Simulación por ordenador y enseñanza de la Física Manuel Belmonte y José L. Rodríguez Illera $C L \& E, 1995,28, p p .63-72$}

Resumen: En este artículo se analiza un programa de simulación que ha sido utilizado para la enseñanza de la Física en secundaria, desde el punto de vista de la utilización de software en situaciones educativas regladas. También se analizan algunos de los aspectos psicopedagógicos más relevantes, así como los cambios didácticos que su uso cotidiano ha conllevado.

Datos sobre los autores: Manuel Belmonte es profesor de Física en el Instituto de Bachillerato Narcís Monturiol de Barcelona. José L. Rodríguez Illera es profesor de la Universidad de Barcelona.

Dirección: Manuel Belmonte. Instituto de Bachillerato Narcís Montoriol. c/ Harmonia, s/n. 08035 Barcelona. José L. Rodríguez Illera. Institut de Ciències de l'Educació. Universitat de Barcelona. Passeig Vall d'Hebron, 171. 08035 Barcelona.

() PERMISOS PARA CITAR O REPRODUCIR EN OTRAS FUENTES: Se pueden citar libremente hasta 500 palabras. Para reproducir una porción de texto mayor, figuras o ilustraciones, se deberá pedir permiso por escrito a la revista, especificando el uso al que se destina el texto. En todos los casos, se deberá citar el copyright de CL\&E. En el caso de artículos o textos que hayan sido a su vez reproducidos en $C L \& E$ los interesados deberán dirigirse tanto a los detentadores del copyright original como a $C L \& E$, en el caso de que se quiera hacer uso de la traducción. FOTOCOPIAS: Para todo lo relacionado con el uso mediante fotocopia del material de esta revista, deberán dirigirse a: CEDRO, $\mathrm{C} /$ José Marañón, 10, 3. Izda. Tel. 5941575 . Fax 4453567 